\title{
A Double Auction Economic Model for Grid Services
}

\author{
Liviu Joita ${ }^{1}$, Omer F. Rana ${ }^{1}$, W. Alex Gray ${ }^{1}$, and John Miles ${ }^{2}$ \\ 1 School of Computer Science and Welsh eScience Centre \\ 1.joita@cs.cardiff.ac.uk \\ 2 School of Engineering, Cardiff University, UK
}

\begin{abstract}
The use of a service-based approach in Grid computing will lead to such services becoming valuable economic commodities. Current economics models on the Internet are concerned with the creation, sale and purchase of commodities (generally information resources), and ways in which buyers and sellers interact in markets for them. Although several initiatives are engaged in the development of Grid technologies, Grid economy issues are yet to be fully addressed. A "Grid market" architecture based on the double auction economic model is proposed. Components of the design, and a prototype of the double auction model framework, which makes use of the Globus and Java CoG toolkits, are subsequently presented.
\end{abstract}

Combining commodity technologies like Web Services with Grid technologies like Globus/UNICORE, has led to the development of Grid Services. This change is significant, as it enables the use and provision of such services on a variety of different platforms and operating systems. The price at which services can be sold depends on factors such as demand, supply and how the market operates. Participants in a Grid services market may be suppliers of computational or storage resources, or consumers as application or data users. We make the following assumptions within our market model: the demand for a particular Grid service is determined by the value users place upon the service, and the price they are willing to pay to obtain it. We also assume that the number of Grid services that are supplied in the market depends on how much suppliers can expect to charge for them, and costs of any existing similar services. The nature of competition amongst suppliers, how they interact with customers, and how the market is regulated play an important role on the pricing of Grid services. Based on the price charged and market rules, a provider has to recover cost and remain active on the market. The economic models and charging rules for Grid computing have to evolve according to market driven forces. A trading mechanism based on the auction economic model for Grid services should provide tools to deliver some overall value (or utility) to users, and it should allow them to express their requirements and overall goals.

The components of a Grid auction system, (referred to as the Grid Market Model Flow) based on a double auction model, are described. In a double 
auction system buyers and sellers are treated symmetrically, with buyers submitting bids and sellers submitting asks. The architecture combines workflow approaches with a resource trading model provided through the double auction system. We focus on the security and auction issues, which differs in focus from Grid Bank [6] and the procurement process (as investigated by others). The main contribution of this paper is the particular architecture for a Grid market, based on the notion of a Virtual Organisation (VO), and a partial implementation that demonstrates our ideas using Java CoG. The COllaborative VIrtual TEams (COVITE) project [8] develops a software tool in which such of VOs can form and evolve and offers also the possibility of developing and integrating instances of Grid markets. The Figure 1(a) shows the PSCD application integrated with the Grid market component. Resources can belong to multiple concurrent VOs, participants may buy or sell their resources (for example CPU time, CPU power and memory in order to host Slave Grid Services of their VOs) to Grid market instances, so the utilisation of their own surplus can be maximised. The paper is organised as follows: Section 1 illustrates related work of auction systems for Grid resources. Section 2 presents the Grid Market Model Flow architecture. Section 2.1 illustrates the double auction Grid economic market architecture scenarios and some implementation. Conclusion and further work follows in Section 3.

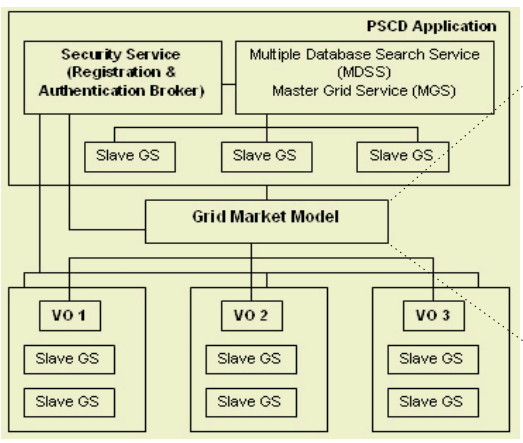

(a)

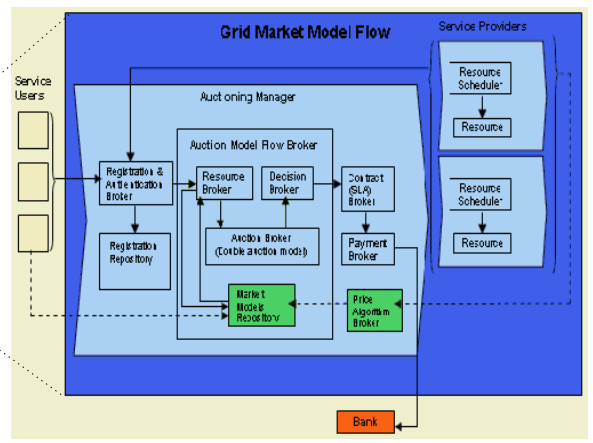

(b)

Fig. 1. Grid Market Module integration within the PSCD application.

\section{Related Work}

Various economic models including microeconomic and macroeconomic principles can be applied when sharing Grid resources in a market. Some of the commonly used economic models include: Commodity Market Model (service providers specify the service price and charge users according to the amount of service they utilise), Posted Price Model (similar to commodity market model except that there are special offers for services from time to time in order to attract more users), Bargain Model (resource providers offer their resources at a price as 
low as possible for use over longer time periods), Auctioning Model (such as those based on e-commerce, e-auctioning, e-marketplace, etc), Tendering/ContractNet Model (based on the contracting mechanism - which governs the agreement between users and service providers for consuming the resources), Cooperative Bartering Model (resources are shared within a community - and members are allowed to barter for resources owned by the community), Monopoly (the case when a single service provider is the only one on the market offering a particular service and therefore dictates the resource price), Market-based control (whereby an 'artificial economy' is established for sharing resources) [2]. In addition to these economic models, different pricing schemes apply on the markets, such as: Flat price model (once the price is established it remains unchanged for a certain period of time), Competitive economic models (such as e-auctioning and e-marketplaces), Usage timing (peak/off-peak - like telephone services), Demand and supply based (prices change as supply and demand change), Foresight-based (based on the ability to model and predict responses by competitors), Loyalty based (special prices for loyal and regular users), Advance agreement/contract based (the price is agreed and a contract established a time before the use of resources) [2]. Some of these economic models and pricing schemes have already been used in different Grid projects to demonstrate their applicability in a Grid economy. In [3] the commodity market based approach to allocated resources is used, where resources are classified into different categories based on their hardware components, network connectivity and operating systems. An algorithm is presented to determine the price of the resources based on the demands of the resources (or bundled resources) and the supply for commodities. This price is based on the tâtonnement process - that is postulated in economic theory to explain price determination in competitive market. In such a scheme the allocation is not changed until the price reaches equilibrium. The initial price is pre-assigned. The resource is allocated on a first come-first serve base.

In [4] two economic models are used: commodities market and the Vickrey auction. First, the commodity market model treats disparate resources as interchangeable commodities. The supply and demand pricing scheme is applied to determine when transactions are completed. Second, the Vickrey (second-price sealed-bid) auction model is used for determining transaction prices for two commodities - the CPU (processor) and storage. In [5] a market based mechanism for trading CPU time is presented, in which the Vickrey (second-price sealedbid) auction and a simple sealed-bid double auction (both buyers and sellers offer a low price and a high price, as well as a rate of change) are implemented.

Economic theory models have generally been used in the context of resource allocation, although the important question about which model is the most appropriate for supporting resource and allocation on the Grid is often not fully addressed. Often it is also difficult to pinpoint the exact model to use in a particular instance - requiring multiple models to co-exist. However, numerous applications of double auctions in electronic commerce, including stock exchange and financial markets, business-to-business commerce, market-trading of equities, commodities etc, have lead to a great deal of interest in fast and effective algorithms of 
the double auction model [8]. This economic model therefore provides a useful approach in the context of resource allocation on the Grid. We make use of this research, and feel that it best represents the exchange of resources within a Grid environment. The double auction model proposed aims to force the market to a theoretical equilibrium price, as there are posted "bids" (buy orders) or "asks" (sell orders) and the auction process rules determine prices. The Grid services have a price based on the demand, supply, and value in the Grid economy. The real-time process auction periodically closes the bidding process and chooses the winners. Members of the Global Grid Forum GESA working group [6] have started to define the protocols and service interfaces needed to support a variety of economic models for the charging of Grid Services using the Open Grid Service Architecture (OGSA). This effort is concentrated on developing an infrastructure to enable the trading of Grid services, as well as defining additional service data and ports needed to describe an economic Grid service. This has led to the definition of a "Chargeable" Grid Service and the Grid Banking Service, as a step to a Market for Computational Services. In [7] a computational economic framework for resource allocation and for regulating supply and demand in a Grid computing environment is presented. Various economic models from the real world market are described for setting the price for Grid resources such as: commodity market, posted price, tender and auction models, as described earlier in this section. The prices of the resources are based on supply and demand, and their perceived value to a user.

The architecture proposed in this paper is complementary to the GESA working group. It also describes an auction mechanism service, in part presented in [7], and shows the interaction with other components in a Grid market. The main focus is on an auction broker (making use of workflow operations) based on the double auction model. The advantage of the double auction model is that it provides a robust process of price discovery. Bidders can initially bid conservatively, and then raise their bids in response to market forces.

\section{Grid Market Model Flow}

A Grid market is a virtual place where Grid services are traded at specified time periods. The concept of a market may be related to that of a "Virtual Organisation" (VO). A typical Grid project often involves many individuals and institutions collaborating for the duration of the project - forming a VO. Each such VO may be an instance of a Grid market in which resources can be traded.

Grid Market Architecture: Components of the Grid Market Model Flow architecture are illustrated in Figure 1(b). A double auction model in which a 'bid' and 'ask' are submitted to a common Grid market any time during the trading period is used. An ask starts at the highest price and is decreased, and a bid starts at the lowest price and is increased. Customers issue a bid for the resources needed, and Grid resources issue an ask with the price and resources offered. If any bid and ask match their requirements and prices, the trade is executed. The Grid Market Model Flow architecture has two types of 
components: Brokers and Data Repositories - Brokers operate on the data in Repositories. The Brokers include a Registration \& Authentication Broker, an Auction Broker, a Contract Broker, and a Payment Broker.

Registration \& Authentication Broker is used for security management. The broker defines a security framework using the Globus Security Infrastructure (GSI) - requiring users to provide a private key and an X.509 certificate to authenticate themselves [1]. GSI provides a single sign-on capability, and supports delegation, via a proxy, to perform the authentication on users and Grid resources' behalf. Further details regarding the full implementation of the Registration \& Authentication Broker can be find in [11]. Auction Model Flow Broker is used for auction management, and comprises of a Resource, Auction and Decision Broker. The Resources Broker is used to access the Market Models Repository for displaying the current active bids. The Resource Broker contains rules which apply to the market, such as: the definition of a bid string, the reserve price for the ask, time period over which an ask is alive on the market, etc. All participants have to agree with the rules defined in the Resource Broker in order to place their bids or asks in a market. The Auction Broker is used for the real-time auction process, while the Decision Broker decides when the auction process will close and a winner chosen. The Decision Broker also contains rules such as: how long a bid or an ask can stay alive, when a bid or an ask are fired, etc. Contract Broker is responsible for managing the Service Level Agreement (SLA) after the transaction is executed. The SLA is part of the contract that guarantees the quality and the performance of Grid services acquired during the auction process by users. An SLA must cover performance parameters, such as: networking parameters (e.g. bandwidth), computational parameters (e.g. number of processors, CPU time, CPU power, storage capacity, etc), the duration for which the Grid service is required, the cost of the transaction, etc. We explore the use of SLAs in other work [9]. Payment Broker is used to charge for the transaction via a Bank [6]. The Contract Broker initiates, and the Payment Broker closes the procurement process. The Bank is an established infrastructure used to transfer money from the account of a service user to a service provider accounts, to pay for Grid services purchased within the auction.

Data repositories include a Market Models Repository, which both service users and providers have access to. This repository contains a list of prices for different services available on the market. Service providers can define a fair price for their resources using the Price Algorithm Broker, and service users can issue a bid for resources with the price which is in demand on the market at that time. The second repository - Registration Repository is used to keep information about all participants on the market.

\section{$2.1 \quad$ Scenarios}

Two Grid economic market architecture case scenarios are presented from the point of view of how the market is defined: first, a common single market for trading resources, and second, a market that contains sub-markets for each type of resources traded. 
Common Single Market: The following resources can be traded in a common single market: CPU time, CPU power, memory, and storage capacity. The auctions will run according to the following high-level protocol:

1. A Service User submits bids with preferences, threshold and expenditure limits (prices) to the Auction Broker.

2. A Service Provider submits their asks with a "reserved" price to the Auction Broker.

3. The Auction Broker updates its price quote every $T$ minute, indicating the current going price(s). A value of 5 minutes for $T$ is used as the default in our system.

4. The Auction Broker uses a schedule to decide for how long a bid and an ask are alive on the market.

The rules defined in the Decision Broker specify when or under what conditions a bid matches an ask, and record the transaction. A bid or an ask become inactive if they do not lead to a transaction within the scheduled time for which they are alive, as specified in the Auction Broker. Both a service user and provider may decide to once again issue a bid and an ask, or to retrieve them from the market.

The bid format is defined as a bid string - representing a willingness to buy or sell the service in an auction. A service user bid string contains a list of bid points in the following format: $\left(\gamma_{j} \tau_{j}, \mathrm{j}=\{1, \ldots, 4\}\right)$, where: $\tau_{i}$ is the bid substring in the following format: $\tau_{i}=\left(t_{i}, p_{i}\right)$. In this bid, parameter 1 represents CPU time, parameter 2 represents CPU power, parameter 3 represents memory, and parameter 4 represents storage capacity. $t_{i}$ represents the type of the Grid service a participant is willing to buy. $p_{i}$ represents the price of the Grid service a participant is willing to pay for. An Auction Broker uses the following rule: a service user is willing to buy services of type $t_{i}$ for a price $p_{i}$, but not bigger than the expenditure limit prices defined when the bid is placed on the market. $\gamma_{i}$ is the preference (threshold) parameter, and lies between 1 to 10 . The threshold has to be chosen when a bid is placed on the market. A service provider bid string contains a list of bid points in the following format: $\left(\tau_{k}, \mathrm{k}=\{1, \ldots, 4\}\right)$, where $\tau_{i}=\left(t_{i}, p_{i}\right)$. Parameter definitions are the same as before. An Auction Broker uses the following rule: a service provider is willing to sell goods of type $t_{i}$ for a price $p_{i}$, but not less than the reserved price defined when the ask is placed on the market. The bid is placed on the market with a threshold. The bid string contains preferences for each service traded on the market.

The following example illustrates the rules: consider a service user bid string of $(10(1 £ 30), 2(2 £ 25), 4(3 £ 35), 7(4 £ 32))$; the threshold is $£ 5$ and the expenditure limit $£ 33$ for each service; as soon as substrings 1 and 4 are fulfilled (because their preferences are bigger than the threshold) then the bid is fired and the transaction recorded; even if substrings 2 and 3 are not fulfilled, or only one of them is fulfilled. Alternatively, consider a service provider ask string of (-(1 $£ 35),-(2 £ 0),-(3 £ 0),-(4 £ 0))$ with a reserved price of $£ 29$. The requirement of the ask is fully fulfilled if the price offered for it is no less than the reserved price of the ask. If the price is less than the reserved price, the ask is fired but the transaction is not recorded. 
Common Market with Multiple Sub-markets: We now consider a common market which contains multiple sub-markets - with each sub-market being used for trading specific services. As before, the common market contains four submarkets: for CPU time, CPU power, memory, and storage capacity. The auction uses the same high-level protocols and assumptions as in the single market. A service user bid format is defined as follows: $\left(\gamma_{i} \tau_{i}\right)$, where $\tau_{i}=\left(p_{i}\right)$, in which $p_{i}$ represents the price of the Grid service. The following rules will apply in the Auction Broker: (1) the service user is willing to buy the good at the auction, for a price $p_{i}$, but not bigger than the expenditure limit price. (2) $\gamma_{i}$ is the preference (threshold), on a scale from 1 to 10 - chosen when a bid is placed on the market. The service provider bid string contains a list of bid points in the following format: $\left(\tau_{i}\right)$, where $\tau_{i}=\left(p_{i}\right)$, in which $p_{i}$ is the selling price of the Grid service. As before, the service provider wants to sell the service at the auction for $p_{i}$, but not less its reserved price.

Consider the following scenario: if a user needs, for example, three out of four services, bids with their preferences may be placed on specific sub-markets, with a threshold for each bid. A transaction is fulfilled as soon as all the bids from sub-markets complete. If we consider the following service: $1=\mathrm{CPU}$ time, $2=\mathrm{CPU}$ power, $3=$ memory, and $4=$ storage capacity, and only 1,2 , and 4 are required, then the bids in specific sub-markets are as follows: for service $1,10 \tau_{i}$; for $2,4 \tau_{i}$; and for $4,6 \tau_{i}$. The threshold $t$ chosen is 5 . As soon as the bids with the $\gamma_{i}>t$ (service 1 and 4 here) have fulfilled their requirements, service user has traded on each sub-market, but for a price not bigger than the limit expenditure price. The transaction is recorded even if the bid with $\gamma_{i}<t$ (service 2 here) is not matching his requirements. Conversely, a service provider's ask is fully fulfilled if the price offered for it is no less than the reserved price of the ask. If the price is less than the reserved price, the ask is fired but the transaction is not recorded.

The prototype uses RedHat Linux 7.3, and Globus 2.2.4. The programming tools are: Java2 SDK Version 1.4.1_01, Java CoG version 1.1., Servlets, and JSP, with an Apache Tomcat Web server. MS-Access database is used as a Market Model Repository hosted by another machine running the WindowsXP operating system. Further development will upgrade the database to MS SQL Server, which is more robust and scalable than MS-Access. The Service Providers may make available their Grid resources and publish them in the Market Repository, as show in Figure 2. The interface to the Market Repository is an XML based Web Service. The Auction Broker can invoke and search the repository using Simple Object Address Protocol (SOAP) messages. The prototype currently supports security and auction brokers only - work is underway to add additional components.

\section{Conclusion and Further Work}

A Grid market architecture based the double auction theory is described. This auction model is the dominant market model for real-world trading of equities, commodities, derivatives, etc. This paper focuses on two Grid market model 


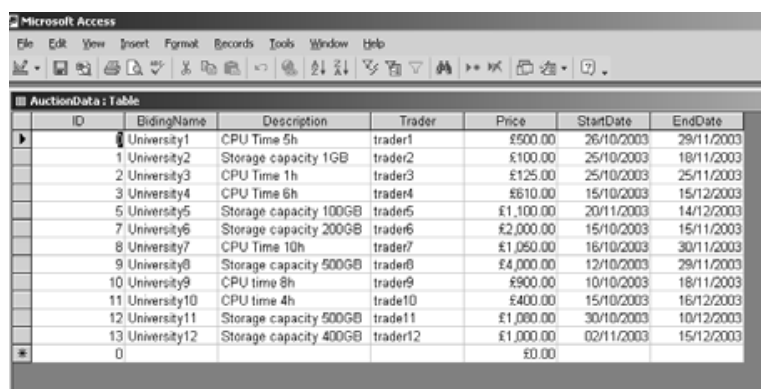

Fig. 2. The Market Repository.

architectures (the designing and development) of an auctioning system, based on the double auction model postulated in the economic literature, used for Grid resource acquisition based on the "supply and demand" principle.

\section{References}

1. Globus Security. See Web site at: www.globus.org/security

2. R. Buyya, D. Abramson, J. Giddy, and H. Stockinger, "Economic Models for Resource Management and Scheduling in Grid Computing". Available at: http://hst.home.cern.ch/hst/publications/emodelsgrid.pdf, 2002.

3. K. Subramoniam, M. Maheswaran, and M. Toulouse, "A Micro-Economic Model for Resource Allocation in Grid Computing Systems", IEEE Canadian Conference on Electrical and Computer Engineer (CCECE '02), May, 2002.

4. R. Wolski, J. S. Plank, T. Bryan, J. Brevik, "G-commerce: Market Formulations Controlling Resource Allocation on the Computational Grid", International Parallel and Distributed Processing Symposium (IPDPS), San Francisco, CA, April, 2001.

5. O. Regev, N. Nisan, "The POPCORN Market - an Online Market for Computational Resources", First International Conference on Information and Computation Economies ICE-98, October 25-28, 1998.

6. S. Newhouse, "GWD-R (draft-ggf-gesa-services-1)", Global Grid Forum, June 2003.

7. R. Buyya, "Grid Economy: A Market Paradigm for Distributed Resource Management and Scheduling for Service Oriented Grid Computing", PhD Thesis, Monash University, Australia, 2002.

8. D. Friedman, J. Rust, "Double Auction Market: Institutions, Theories, and Evidence", Perseus Publishing, 1993.

9. R. Al-Ali and O. Rana and D. Walker and S. Jha and S. Sohail, "G-QoSM: Grid Service Discovery Using QoS Properties", Computing and Informatics Journal, Special Issue on Grid Computing, Vol. 21, No.4, pp 363-382, 2002.

10. COVITE project Web site: http://www.wesc.ac.uk/projectsite/covite/

11. L. Joita, O. Rana, P. Burnap, J. S. Pahwa, A. Gray, J. Miles, "A Grid-Enabled Security Framework for Collaborative Virtual Organisations", 5th IFIP Working Conference on Virtual Enterprises (PRO-VE), Toulouse, France, 22-27 August 2004 (to appear). 